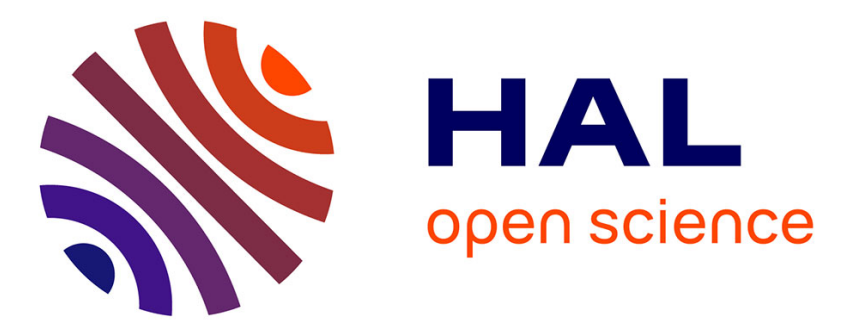

\title{
Multi-orbital kinetic effects on charge ordering of frustrated electrons on the triangular lattice
}

C. Février, Simone Fratini, A. Ralko

\section{To cite this version:}

C. Février, Simone Fratini, A. Ralko. Multi-orbital kinetic effects on charge ordering of frustrated electrons on the triangular lattice. Physical Review B: Condensed Matter and Materials Physics (1998-2015), 2015, 91 (24), pp.245111. 10.1103/PhysRevB.91.245111 . hal-01219133

\section{HAL Id: hal-01219133 \\ https://hal.science/hal-01219133}

Submitted on 1 Feb 2019

HAL is a multi-disciplinary open access archive for the deposit and dissemination of scientific research documents, whether they are published or not. The documents may come from teaching and research institutions in France or abroad, or from public or private research centers.
L'archive ouverte pluridisciplinaire HAL, est destinée au dépôt et à la diffusion de documents scientifiques de niveau recherche, publiés ou non, émanant des établissements d'enseignement et de recherche français ou étrangers, des laboratoires publics ou privés. 


\title{
Multiorbital kinetic effects on charge ordering of frustrated electrons on the triangular lattice
}

\author{
C. Février, S. Fratini, and A. Ralko \\ CNRS, Institut NEEL, F-38042 Grenoble, France \\ and Université Grenoble Alpes, Institut NEEL, F-38042 Grenoble, France
}

(Received 19 March 2015; revised manuscript received 20 May 2015; published 8 June 2015)

\begin{abstract}
The role of the multiorbital effects on the emergence of frustrated electronic orders on the triangular lattice at half filling is investigated through an extended spinless fermion Hubbard model. By using two complementary approaches, unrestricted Hartree-Fock and exact diagonalizations, we unravel a very rich phase diagram controlled by the strength of both local and off-site Coulomb interactions and by the interorbital hopping anisotropy ratio $t^{\prime} / t$. Three robust unconventional electronic phases, a pinball liquid, an inverse pinball liquid, and a large-unit-cell $\sqrt{12} \times \sqrt{12}$ droplet phase, are found to be generic in the triangular geometry, being controlled by the band structure parameters. The latter are also stabilized in the isotropic limit of our microscopic model, which recovers the standard SU(2) spinful extended single-band Hubbard model.
\end{abstract}

DOI: 10.1103/PhysRevB.91.245111

PACS number(s): 71.10.Hf, 73.20.Qt, 71.30.+h, 74.70.Kn

\section{INTRODUCTION}

In analogy with frustrated spin systems, frustration of the charge interactions by the triangular-lattice geometry constitutes a favorable playground for the emergence of novel phases. In this context, the pinball liquid (PL) was proposed a decade ago [1] as an original Coulomb-induced charge-ordered metallic phase in the framework of the extended Hubbard model. This phase has no classical equivalent and takes advantage of quantum fluctuations in order to lift the massive degeneracy of interacting electrons on quarter-filled triangular lattices in the classical limit. While the existence of the PL is now a consolidated fact, as it has been demonstrated by several complementary techniques and approximations [2-7], such an interesting electronic phase has not been observed experimentally in the $\theta$-(BEDT-TTF $)_{2} X$ materials for which it was originally predicted. This can be attributed to the presence of other competing effects not considered in the idealized theoretical descriptions, most notably deviations from a perfectly isotropic triangular lattice, the interaction with the lattice degrees of freedom [8], and the presence of long-range tails of the Coulomb repulsion beyond nearest neighbors $[9,10]$, which all favor insulating stripe-ordered states.

There exist other classes of materials with layered triangular lattices and sizable electronic interactions which do present interesting charge-ordered phases whose origin is not fully understood. These include transition-metal oxides such as the layered cobaltates $\mathrm{Na}_{x} \mathrm{CoO}_{2}$, which exhibit complex electronic patterns which can be tuned by electron doping [11-14], and the triangular nickelates $\mathrm{AgNiO}_{2}[15,16]$ and $\mathrm{Ag}_{2} \mathrm{NiO}_{2}$ [17], which show a threefold ordered metallic phase with anomalous metallic properties. Another interesting class is that of transition-metal dichalcogenides. In $1 T-\mathrm{TaS}_{2}$, for example, the ordered state displays a marked Mott character induced by charge modulations with a large periodicity of $\sqrt{13} \times$ $\sqrt{13}[18,19]$, and various other periodicities are found in other compounds. What all these materials have in common is that electrons live in bands constructed from $d$ atomic orbitals. Bridging the ideas of frustrated charge order from their initial domain of application (single-band, layered organic conductors) to such $d$-electron compounds requires us to account for the presence of multiple bands and to move to electron densities not restricted to one-quarter filling.

Multiband effects come in two different kinds. The first is related to interactions that are present within the $d$-electron manifold already at the atomic level, most notably the onsite Hund interactions acting on the magnetic degrees of freedom [20]. These are known to favor the emergence of high-spin states and have been shown to strongly enhance the effects of electronic correlations [21-23]. Their ability to stabilize a PL phase with unconventional metallic properties at a filling of one electron per site has been explored very recently [24]. The second type of multiband effect, which can also lead to novel and original properties, is of kinetic origin and has to do with the microscopic form and symmetry of the interatomic electron transfers. One remarkable example is the hidden kagome symmetry and flat bands which have been pointed out in the layered cobaltates [25] and which could be related to the experimentally observed kagome order in these compounds [14,26].

The purpose of this paper is to study how such multiorbital kinetic effects influence the frustrated charge order on the triangular lattice. To this aim we employ an atomistic tight-binding description [27] and set up a two-orbital extended Hubbard model where the band structure can be tuned systematically as a function of the microscopic transfer parameters. Since our main focus is the exploration of novel charge-ordered phases, it is a good starting point to resort to a spinless electron description. This approach has been shown to capture the correct ordering patterns realized in single-band models [1,5] in the limit of strong local Coulomb repulsion, where the magnetic energy scales are typically much smaller than the ones controlling charge ordering. It has also been successfully used to study charge ordering in the context of multiband models for correlated oxides [28] in the ferromagnetic state.

Our results, obtained here at half filling via unrestricted Hartree-Fock (UHF) mean-field theory and exact diagonalization (ED) on small clusters, show the emergence of a rich panel of charge and orbitally ordered phases. Most notably, we find three robust unconventional charge-ordered phases, whose occurrence can be tuned by varying the multiorbital band structure parameters. Two of these phases have peculiar properties since a fraction of the electrons forms a charge order 
with a threefold symmetry breaking, while the other fraction is free to move on the remaining sites of the lattice, forming a honeycomb structure. These phases are called the pinball liquid (originally found in quarter-filled lattices and obtained here at half filling) and the inverse pinball liquid (which can be viewed as the dual to the PL). The third unexpected phase found in this work is the large-unit-cell $\sqrt{12} \times \sqrt{12}$ droplet phase, also found in the isotropic limit where our model reduces to the spinful extended Hubbard model on the triangular lattice, where it was overlooked in previous studies. Such phases could be of relevance to a variety of triangular $d$-band electron systems such as the cobaltates, nickelates, and dichalcogenides.

This paper is organized as follows. The microscopic model and the two different methods of solution are described in Sec. II along with their respective advantages. The phase diagrams obtained from both methods upon varying the interaction parameters and the multiorbital band structure parameters are presented in Sec. III, together with a detailed description and characterization of the different ordered phases. Our main results are summarized in Sec. IV.

\section{MODEL AND METHODS}

\section{A. Spinless two-orbital extended Hubbard model}

To explore the influence of multiorbital effects on Coulombdriven charge ordering on the triangular lattice we write the following spinless two-orbital extended Hubbard model:

$$
\begin{aligned}
H= & -\sum_{\langle i j\rangle, \tau \tau^{\prime}} t_{i j}^{\tau \tau^{\prime}} d_{i \tau}^{\dagger} d_{j \tau^{\prime}}+\text { H.c. }+\tilde{U} \sum_{i} n_{i \uparrow} n_{i \downarrow} \\
& +V \sum_{\langle i j\rangle}\left(n_{i \uparrow}+n_{i \downarrow}\right)\left(n_{j \uparrow}+n_{j \downarrow}\right),
\end{aligned}
$$

which we study at a density of one electron per site. The first term describes $d$ electrons (creation and annihilation operators $d_{i \tau}^{\dagger}$ and $d_{i \tau}$ ) moving with transfer integrals $t_{i j}^{\tau \tau^{\prime}}$ which depend on both the orbital type and on the orientation of the bond $(i, j)$ on the triangular lattice. The second term is an effective onsite Hubbard repulsion between electrons on different orbitals, with $n_{i, \tau}=d_{i \tau}^{\dagger} d_{i \tau}$ being the local electron density on orbital $\tau$. The third term is the Coulomb repulsion between electrons on neighboring sites, which constitutes the driving force for charge ordering. Note also that in the electrostatic limit, if the presence (absence) of an electron at site $i$ is interpreted as a spin up (down), we immediately see that this last term is responsible for the presence of geometrical frustration on the charge degrees of freedom on the triangular lattice, in analogy with magnetic systems.

Besides its fundamental interest per se, such a two-band description applies to actual materials with complete $t_{2 g}$ and partially filled $e_{g}$ shells. In $\mathrm{AgNiO}_{2}\left(t_{2 g}^{6} e^{1}{ }_{g}\right.$ configuration, formal valence $\mathrm{Ni}^{3+}$ ), for example, the orbitals are split between an $e_{g}$ doublet occupied by one electron and a completely filled $t_{2 g}$ triplet that can be neglected to a first approximation by virtue of the large crystal-field gap of $\sim 2 \mathrm{eV}$ [29-31]. Since the twofold $e_{g}$ orbitals $\left|d_{3 z^{2}-r^{2}}\right\rangle$ and $\left|d_{x^{2}-y^{2}}\right\rangle$ form a pseudospin-1/2, we label them, respectively, as $|\uparrow\rangle$ and $|\downarrow\rangle$ and denote the corresponding Pauli matrices by $\tau^{i}$

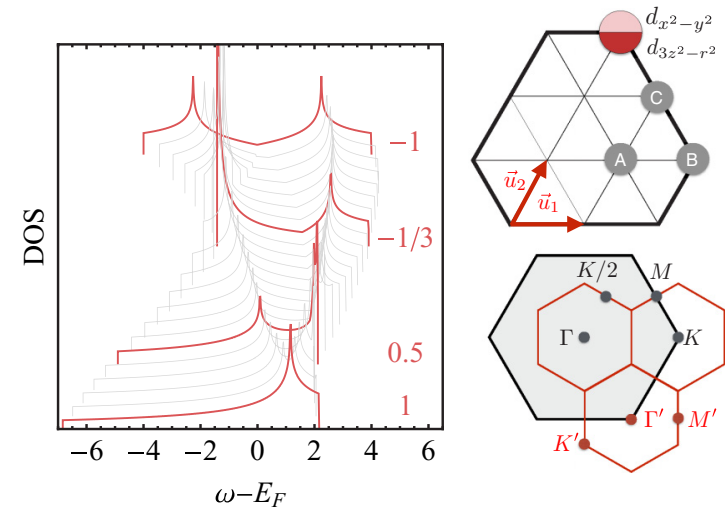

FIG. 1. (Color online) Evolution of the density of states of the noninteracting system with respect to the $t^{\prime} / t$ ratio. The sketches illustrate (top) the triangular lattice with two orbitals per site and sublattices A, B, and C, corresponding to threefold charge ordering, and (bottom) the main symmetry points in the original and reduced Brillouin zone for three-sublattice ordering.

with $i=1,2,3$. The transfer integrals along the lattice vectors $\vec{u}_{1}, \vec{u}_{2}$, and $\vec{u}_{3}=\vec{u}_{2}-\vec{u}_{1}$ sketched in Fig. 1 can be expressed in terms of two independent parameters $t$ and $t^{\prime}$ as [27,29,32]

$$
t_{\vec{u}_{1}}=\left(\begin{array}{cc}
t & 0 \\
0 & t^{\prime}
\end{array}\right), \quad t_{\vec{u}_{2}}=\left(\begin{array}{ll}
t_{2} & t_{3} \\
t_{3} & t_{4}
\end{array}\right), \quad t_{\vec{u}_{3}}=\left(\begin{array}{cc}
t_{2} & -t_{3} \\
-t_{3} & t_{4}
\end{array}\right),
$$

with $t_{2}=\left(t+3 t^{\prime}\right) / 4, t_{3}=\sqrt{3}\left(t-t^{\prime}\right) / 4$, and $t_{4}=\left(3 t+t^{\prime}\right) / 4$. In full generality we take $t^{\prime} / t$ in the interval $[-1,1]$ (all values outside this interval amount to interchanging orbitals $a$ and $b$ ) and set $t$ as the energy unit. Note that for $t^{\prime}=t$ the kinetic term reduces to two independent instances of the triangular isotropic lattice, and the model becomes analogous to the single-band spinful extended Hubbard model.

The effective on-site Hubbard repulsion term in Eq. (1) describes the interaction between electrons on different orbitals. It obviously has direct relevance to the study of ferromagnetically ordered states [28], in which case only one spin species is present and two electrons necessarily occupy two different orbitals. In a more general context, the present spinless model can be viewed as an approximation to tackle the strongly interacting (i.e., strong Hubbard repulsion and strong Hund coupling) limit of the two-band spinful model introduced in Ref. [24]. Following standard notations [20,23], the interaction energy of aligned-spin configurations on a site is $\tilde{U}=U-3 J_{H}$, where $J_{H}$ is the Hund exchange coupling and $U$ is the intraorbital Hubbard repulsion. Such configurations are favored (in other words, Hund's coupling favors high-spin states) because all other configurations have energies $U-2 J_{H}$ and higher. When $J_{H}$ approaches $U / 3$, the condition $U-$ $2 J_{H} \gg \tilde{U}$ makes it is possible to restrict the system to states with aligned spins since these become energetically more favorable, projecting out all other high-energy configurations. Spinless fermions constitute a reasonable approximation to this projection for those aspects of charge ordering which do not involve the magnetic degrees of freedom. Finally, a notable advantage of the spinless model is that it allows us to cross-check the mean-field results via the use of ED techniques 
which would be impossible if we considered the full orbital and spin character in the model.

We mention here that although related multiband models for charge and orbital ordering on the triangular lattice have been studied in recent years, how multiorbital kinetic terms affect frustrated charge ordering (in particular the pinball liquid) remains an open question. Vernay et al. [29], for example, studied the evolution of orbital ordering as a function of $t^{\prime} / t$ in a spinful model via both mean-field and exact diagonalization, but they did not consider the charge ordering induced by the intersite repulsion $V$. Uchigaito et al. [32] performed a mean-field analysis of the effects of both the Hund and Jahn-Teller couplings on the electron ordering as a function of $t^{\prime} / t$; however, because the repulsion $V$ was not included in the model, no pinball-liquid phase was found at realistic values of the Hubbard repulsion $U$, which is at odds with the experimental observations in $\mathrm{AgNiO}_{2}$. The question has also been addressed from an ab initio point of view [33], including both local and nonlocal interaction effects, but without providing systematic studies as a function of the microscopic Hamiltonian parameters. Finally, multiband effects on charge ordering on the triangular lattice have been studied via both UHF and dynamical mean-field theory in Ref. [24], but only the fully isotropic limit $t^{\prime}=t$ was explored.

\section{B. Methods}

To solve Eq. (1) we have used the UHF and ED methods, which are both defined on the site basis. There are two types of clusters with periodic boundary conditions on the triangular geometry which respect all the symmetries of the infinite lattice (translations and point-group symmetries). In terms of the basis vectors $\vec{u}_{1}$ and $\vec{u}_{2}$, they are defined by two vectors,

$$
\begin{aligned}
& \vec{T}_{1}=l \vec{u}_{1}+m \vec{u}_{2}, \\
& \vec{T}_{2}=-m \vec{u}_{1}+(l+m) \vec{u}_{2},
\end{aligned}
$$

with $l$ or $m=0$ for regular clusters (considered here for the UHF computations) and $l=m$ for tilted ones used for the ED. The regular clusters have a number of sites $N=l^{2}$, while it is $N=3 l^{2}$ for the tilted lattices. In order to allow all relevant symmetry breakings, we have considered lattices for which the number of sites is always a multiple of 12 .

In UHF, the interaction terms $n_{i \uparrow} n_{j \downarrow}$ are decoupled as a Hartree term $\left\langle n_{i \uparrow}\right\rangle n_{j \downarrow}+n_{i \uparrow}\left\langle n_{j \downarrow}\right\rangle-\left\langle n_{i \uparrow}\right\rangle\left\langle n_{j \downarrow}\right\rangle$ and a Fock term $\left\langle d_{i \uparrow}^{\dagger} d_{j \downarrow}\right\rangle d_{j \downarrow}^{\dagger} d_{i \uparrow}+d_{i \uparrow}^{\dagger} d_{j \downarrow}\left\langle d_{j \downarrow}^{\dagger} d_{i \uparrow}\right\rangle-\left\langle d_{i \uparrow}^{\dagger} d_{j \downarrow}\right\rangle\left\langle d_{j \downarrow}^{\dagger} d_{i \uparrow}\right\rangle$. The sets $\left\langle n_{i \tau}\right\rangle$ and $\left\langle d_{i \tau}^{\dagger} d_{j \tau^{\prime}}\right\rangle$, for $i$ and $j \in[1, N]$, are computed from the wave functions in the single-electron basis. A self-consistent loop is performed starting from different initial trial states (homogeneous, random, charge, and/or orbitally ordered) until a fixed point is reached. The result with the lowest energy is selected among the converged states. The method is free from local constraints and ad hoc symmetrizations of the solution; that is, no particular form of the ground state is assumed. This allows us to obtain the most general ordered states of the model in an unbiased way and has been proven very successful in predicting novel phases in related models [24,34].

Also due to the absence of constraints, the resulting mean-field Hamiltonian does not necessarily commute with $\tau^{2}$ and $\tau^{z}$, which are, respectively, the total orbital pseudospin operator and its $z$ component. We notice that the Fock terms of the Hubbard interaction can be recast in terms of the ladder pseudospin operators $\tau^{ \pm}$as $\left\langle\tau_{i}^{+}\right\rangle \tau_{i}^{-}+\left\langle\tau_{i}^{-}\right\rangle \tau_{i}^{+}$, allowing for the extraction of the average orbital components $\left\langle\tau^{x}\right\rangle,\left\langle\tau^{y}\right\rangle$, and $\left\langle\tau^{z}\right\rangle$. This is particularly useful in order to characterize, in addition to the charge symmetry breaking, solutions having an orbital order such as the $120^{\circ}$ phase already observed in the Hubbard model at large $U[35,36]$. To avoid spurious solutions and to obtain smooth convergences, we have employed here a finite-temperature version of the UHF, checking for all our results that the low-temperature regime was reached and no thermal fluctuations remained. Typically, this is always achieved for an inverse temperature $\beta=1 / 50 t$. Finally, we have performed a systematic size-scaling analysis for clusters up to $72 \times 72$ sites in order to identify transition lines in the thermodynamic limit.

For the ED calculations, we have taken the largest available cluster fulfilling all the symmetry requirements mentioned above, namely, the 12-site tilted cluster $(l=m=2$, as depicted in Fig. 1). Since the Hamiltonian does not preserve the orbital flavor, one has to consider, for a fixed number of particles, all the orbital sectors. In addition, due to the structure of the hopping matrix, as soon as $t^{\prime} \neq t$, the three directions of the lattice become inequivalent, hence breaking the $C_{6} v$ point-group symmetry. The system can thus be block diagonalized simultaneously for $k$ points compatible with the remaining $C_{2}$ symmetries, which contain only two generators, $I d$ and $R_{\pi}$, the rotation of an angle of $\pi$ around the $z$ axis. This is true for only points $\Gamma$ and $M ; K$ and $K / 2$ are not compatible with such symmetries (see the corresponding Brillouin zone in Fig. 1). In a given $k$ sector, the ground state (GS) can then be either symmetric (referred to as $A$ ) or antisymmetric $\left(A_{p}\right)$ against the $C_{2}$ symmetries. This will be used in the interpretation of the ED phase diagram.

For both UHF and ED we have identified the different phases via the following quantities: (i) the charge-charge correlations $C(k)=(1 / N)\left\langle\psi_{0}|\rho(-k) \rho(k)| \psi_{0}\right\rangle$, with $\rho(k)$ being the Fourier transform of the total on-site density operator $n_{i \uparrow}+n_{i \downarrow}$, (ii) the orbital-orbital correlations $T(k)=$ $(1 / N)\left\langle\psi_{0}\left|\tau^{z}(-k) \tau^{z}(k)\right| \psi_{0}\right\rangle$, where $\tau^{z}$ is the $z$ component of the pseudospin-orbital operator, and (iii) the average double occupation $D=(1 / N)\left\langle\psi_{0}\left|\sum_{i} n_{i \uparrow} n_{i \downarrow}\right| \psi_{0}\right\rangle$. Finally, we also computed the spectral function

$$
A_{\tau}(k, \omega)=\frac{-1}{\pi} \operatorname{Im}\left\langle\psi_{0}\left|d_{k \tau} \frac{1}{\omega-H+i \eta} d_{k \tau}^{\dagger}\right| \psi_{0}\right\rangle
$$

within the UHF approach to analyze the reconstruction of the band structures and Fermi surfaces.

\section{RESULTS}

\section{A. Noninteracting limit}

Figure 1 reports the evolution of the noninteracting density of states (DOS) as a function of $t^{\prime} / t$. For $t^{\prime} / t=1$, the model reduces to two independent instances of the triangular lattice. As soon as $t^{\prime} \neq t$, however, the electronic dispersion separates into two nondegenerate bands. Correspondingly, the logarithmic singularity in the DOS of the triangular lattice splits into two peaks, and Dirac cones appear in the band 
structure at the $K$ points. Although the DOS never vanishes at the Dirac points, due to the simultaneous presence of other bands at the same energy, these can be identified in Fig. 1 by the kinks located between the two Van Hove singularities. A Lifshitz transition occurs for $t^{\prime} \simeq 0.43 t$ where the lowest of the two peaks in the DOS crosses the Fermi energy, and one of the two bands changes character from electronlike to holelike. The band structure eventually becomes particle-hole symmetric for $t^{\prime}=-t$, in which case the Dirac point falls at the band center. In this case, the system is a semimetal with two parabolic bands touching at the $\Gamma$ point and Dirac cones at the $K$ points.

\section{B. Unrestricted Hartree-Fock}

The phase diagram obtained in the $(\tilde{U}, V)$ plane for $t^{\prime} / t=0.5$ from UHF is reported in Fig. 2(a). We choose this representative value of $t^{\prime} / t$ because the corresponding noninteracting DOS qualitatively reproduces the main features calculated by DFT-LDA for $\mathrm{AgNiO}_{2}$ [31]. To explore all the possible regimes of the model, we also display in Fig. 2(b) the phase diagram in the $\left(t^{\prime}, V\right)$ plane at a fixed value of $\tilde{U}=1$. We recall here that such a moderate $\tilde{U}$ actually stands for a local Hubbard repulsion $U$ which is considerably larger than this value. As discussed after Eq. (1), $\tilde{U}$ is an effective parameter
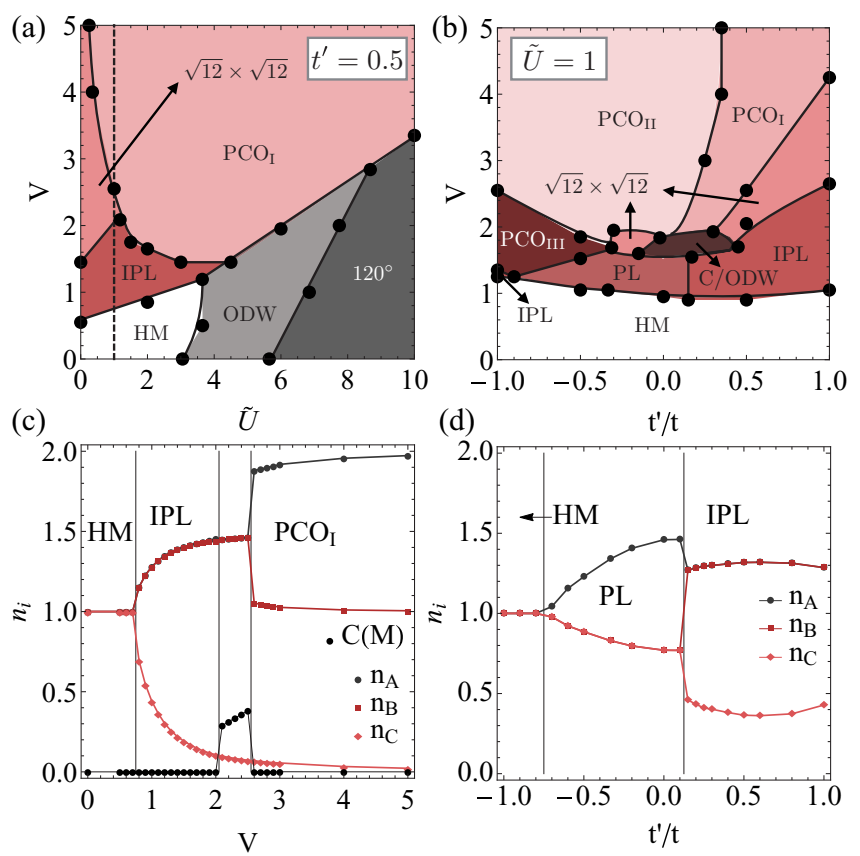

(d)

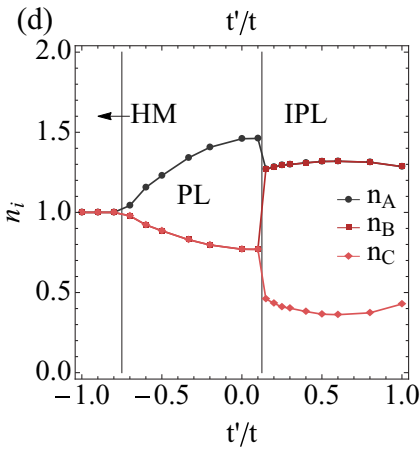

FIG. 2. (Color online) (a) Phase diagram obtained from UHF at fixed $t^{\prime}=0.5 t$. The phases denoted by HM and IPL are metallic, and all the other phases are insulating. IPL is charge ordered, $\mathrm{PCO}_{\mathrm{I}}$ and $\sqrt{12} \times \sqrt{12}$ are charge and orbitally ordered, and ODW and $120^{\circ}$ are orbitally ordered. (b) Phase diagram at fixed $\tilde{U}=1$ as a function

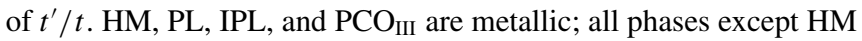
are charge ordered. (c) Evolution of the three sublattice densities $n_{A}, n_{B}, n_{C}$ and charge correlations $C(M)$ along the constant $\tilde{U}$ line shown in (a) [the scale of $C(M)$ has been multiplied by 10]. For the $\sqrt{12} \times \sqrt{12}$ phase, $n_{A}, n_{B}, n_{C}$ are defined as the averages over the four nonequivalent sublattices in the new unit cell. (d) Sublattice densities as a function of $t^{\prime} / t$ for fixed $\tilde{U}=1$ and $V=1.1$.
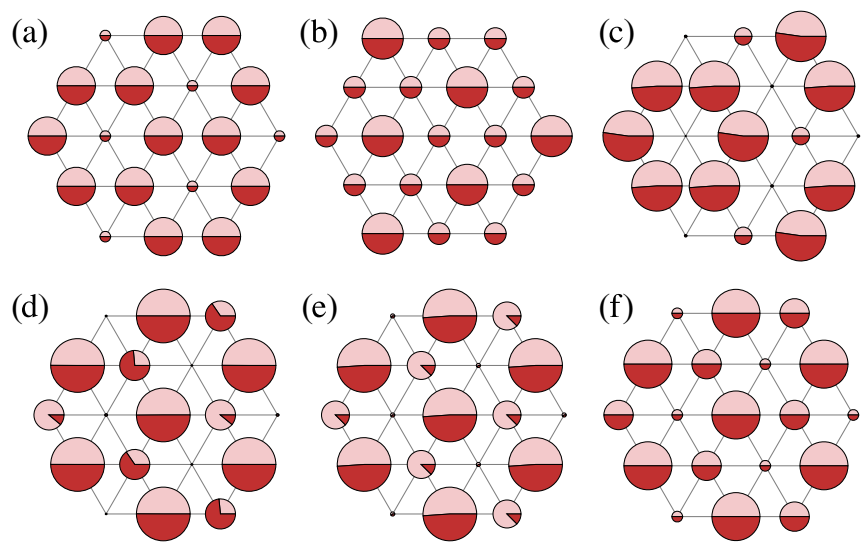

FIG. 3. (Color online) Typical charge- and orbital-density snapshots in the different charge-ordered phases realized in the model: (a) IPL, (b) PL, (c) $\sqrt{12} \times \sqrt{12}$, (d) $\mathrm{PCO}_{\mathrm{I}}$, (e) $\mathrm{PCO}_{\mathrm{II}}$, and (f) $\mathrm{PCO}_{\mathrm{III}}$. The radius of the disks is proportional to the charge density on the sites, and the light and dark fillings correspond to the partial orbital densities.

which is related to the microscopic interaction parameters in the $d$-electron manifold via the equality $\tilde{U}=U-3 J_{H}$. For a choice of the Hund coupling $J_{H}=0.25 U$, for example, the value $\tilde{U}=1$ corresponds to $U=4$, a value appropriate to moderately correlated materials.

As we show below, a rich variety of phases is found and can be classified into charge ordered (at large $V$ ) and charge homogeneous (at low $V$ ). Among the latter, several types have been identified in the model, whose charge and orbital density patterns are depicted in Fig. 3. These are denoted as inverse pinball liquid (IPL), PL, pinball charge order (PCO), and the $\sqrt{12} \times \sqrt{12}$ droplet phase. Supplementing the real-space snapshots, Fig. 2(c) conveniently reports the evolution of the local charge densities as a function of $V$ along the vertical line shown in Fig. 2(a) corresponding to $\tilde{U}=1$. An analogous scan is presented in Fig. 2(d) for the $t^{\prime}$ dependence at fixed $V=1.1$.

(a) Inverse pinball liquid. The IPL order develops in the whole region $t^{\prime} / t>0.1$ upon increasing the intersite repulsion $V$ from the homogeneous metal phase (HM) for sufficiently low values of $\tilde{U}$ [Figs. 2(b) and 2(c)]. This phase exhibits charge order with a three-sublattice structure (A, $\mathrm{B}, \mathrm{C})$. The charge density separates into charge-rich sites forming a honeycomb lattice (balls, density $n_{A}=n_{B}=1+\delta$, with $\delta$ being the charge disproportionation) and charge-poor sites (pins, density $n_{C}=1-2 \delta$ ) located on the remaining triangular sublattice (we conventionally label the sublattice densities in descending order, $n_{A}>n_{B}>n_{C}$ ). The charge disproportionation progressively increases with $V$ towards the maximum allowed value $\delta=1 / 2$ corresponding to a fully depleted charge-poor sublattice $\left(n_{C}=0\right.$ and, correspondingly, $n_{A}=n_{B}=3 / 2$ ). There is no orbital polarization in either the charge-rich or charge-poor sublattices.

The IPL is metallic due to the presence of itinerant carriers (balls) on the charge-rich honeycomb network. Figure 4(a) illustrates the Fermi surface (FS) obtained in this phase $(V=$ $\left.0.9, \tilde{U}=1, t^{\prime}=0.5\right)$, which clearly shows the existence of holelike carriers around the $K$ points of the original Brillouin 
(a)

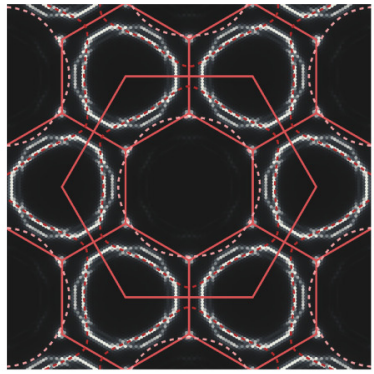

(b)

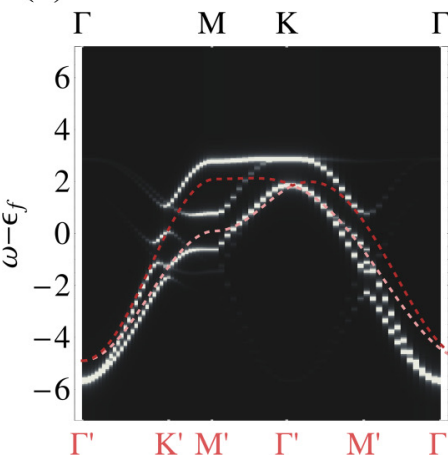

(c)

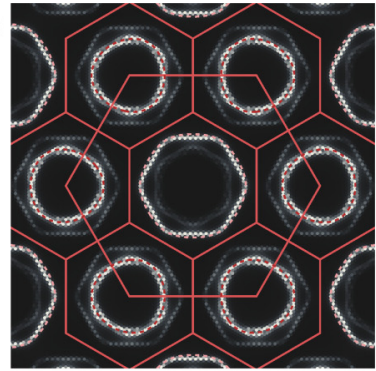

(d)

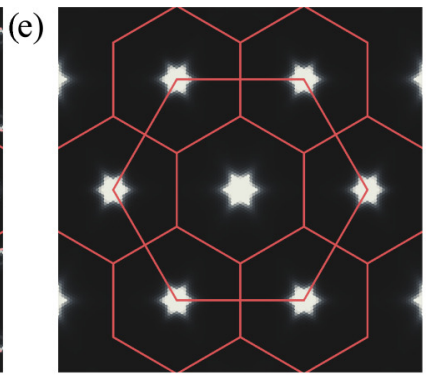

(f)
$\Gamma \quad \Gamma \quad \mathrm{M} \quad \mathrm{K}$

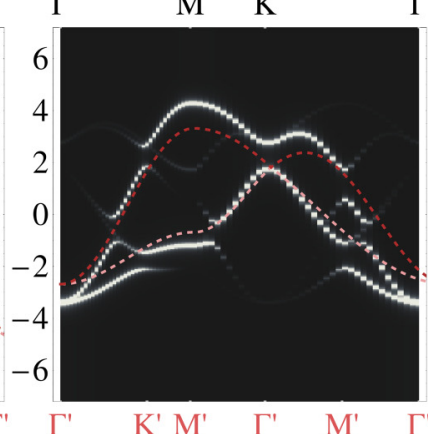

$\Gamma$

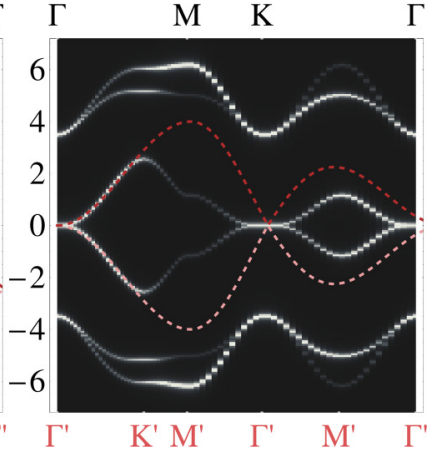

FIG. 4. (Color online) Fermi surfaces and spectral functions for the threefold metallic charge-ordered phases, obtained from Eq. (2) with a Lorentzian broadening $\eta=0.02$ on $72 \times 72$ site lattices: (a) and (b) $\operatorname{IPL}\left(V=0.9, \tilde{U}=1, t^{\prime}=0.5\right)$, (c) and $(\mathrm{d}) \operatorname{PL}\left(V=1.1, \tilde{U}=1, t^{\prime}=0\right)$, and (e) and (f) $\mathrm{PCO}_{\mathrm{III}}\left(V=2, \tilde{U}=1, t^{\prime}=-1\right)$. The solid lines in the top panels show the original and reduced Brillouin zone, whose symmetry points are labeled in Fig. 1. The dashed lines are the Fermi surfaces and band structures in the noninteracting limit.

zone (large hexagon), resulting from the folding of one of the noninteracting bands (shown as dashed lines). Small pockets can also be seen around the corners of the reduced Brillouin zone (small hexagons). These are remnants of the second band of the noninteracting system, which at this value of $t^{\prime}$ crosses the reduced Brillouin zone very close to its corners (denoted as $K^{\prime}$ points) and therefore folds into closed pockets of trigonal shape. The origin of the large hole FS and the small trigonal pockets can also be clearly seen by comparing the spectral function $A(k, \omega)$ illustrated in Fig. 4(b) with the dispersion of the two noninteracting bands (dashed lines).

(b) Pinball liquid. When $t^{\prime} / t<0.1$, the IPL $\frac{3}{2}-\frac{3}{2}-0$ charge pattern shown in Fig. 3(a) is replaced by the PL, with a $2-\frac{1}{2}-\frac{1}{2}$ pattern as illustrated in Fig. 3(b). This phase is dual to the IPL in the sense that the roles of the charge-rich and charge-poor sublattices are interchanged. We identify this phase with the original PL of Hotta and coworkers [1] because, from the point of view of the electronic densities $\left(n_{A}=2, n_{B}=n_{C}=\right.$ $1 / 2$ ), it can be viewed as two realizations of the PL phase found at quarter filling, one per each orbital character (the PL at quarter filling has $\left.n_{A}=1, n_{B}=n_{C}=1 / 4\right)$. Because of orbital-orbital interactions, however, the two realizations are not independent, and the present PL can occur for only small or moderate values of $\tilde{U} \lesssim 5$. This can be contrasted with the quarter-filled case, where a strong Hubbard term is required to stabilize the PL [4-6].

The phase diagram in Fig. 2(b) shows that the selection between the PL order and its dual IPL is entirely governed by kinetic effects. This can be understood by observing that the electrostatic energies of the PL and IPL are formally equal and do not depend on the sign of the charge disproportionation $\delta$, $E_{I P L}=E_{P L}=3 V\left(1-\delta^{2}\right)$.
Similar to the IPL, metallic behavior arises in the PL from the motion of electrons living on the honeycomb network. At this value of $t^{\prime}$, the noninteracting FS (dashed line) is composed of a large electronlike surface around the $\Gamma$ point and smaller holelike pockets around the $K$ points. Contrary to the IPL shown previously, however, the overall shape of the FS is only weakly affected by charge ordering of the PL type because the original Fermi pockets do not cross the boundaries of the reduced Brillouin zone [Figs. 4(c) and 4(d)].

(c) Pinball charge order. At large $V$, the system undergoes further charge ordering, stabilizing a 2-1-0 charge pattern termed pinball charge order [Figs. 3(d)-3(f)]. Simple electrostatic arguments predict that the PCO is stabilized for $V>\tilde{U} / 3$, which nicely agrees with the numerical results at large $\tilde{U}$ and $V$ [see Fig. 2(a)]. The threefold disproportionation splits the electronic dispersion into three separate bands per orbital state. Counting the occupied states leaves us with a central half-filled band, which should lead, in principle, to a metallic behavior. We find instead that the PCO phase found in the whole region $t^{\prime} / t \gtrsim 0.1$, denoted PCO I in Figs. 2(a) and 2(b), is insulating [see the spectral function in Fig. 5(a)]. This is ascribed to the presence of a spiral $120^{\circ}$ orbital order on the singly occupied $B$ sites, caused by the local interaction $\tilde{U}$, as seen in the snapshot in Fig. 3(d). Other orbital orderings on the $B$ sublattice are possible within the present 2-1-0 charge arrangement upon varying $t^{\prime} / t$ [Fig. 2(b)]. These are $\mathrm{PCO}_{\mathrm{II}}$ for $t^{\prime} / t \lesssim 0.1$ and large $V$ [uniformly polarized B sites, Fig. 3(e)] and $\mathrm{PCO}_{\text {III }}$ for $t^{\prime} / t \lesssim 0.1$ and low $V$ [unpolarized B sites, Fig. 3(f)]. $\mathrm{PCO}_{\mathrm{II}}$ is also an insulator because the ferro-orbital polarization is sufficient to split the narrow half-filled band at the Fermi energy into two separate components [Fig. 5(b)]. Note that this is the only phase in the 

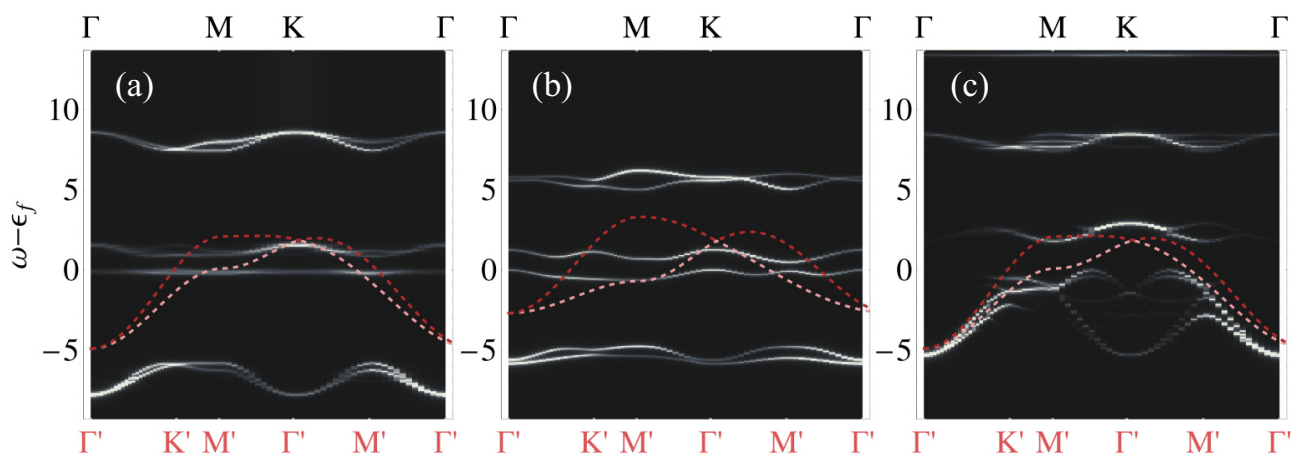

FIG. 5. (Color online) Spectral functions for the insulating charge-ordered phases: (a) $\mathrm{PCO}_{\mathrm{I}}\left(t^{\prime} / t=0.5, \tilde{U}=1, V=2.6\right)$, (b) PCO $\left(t^{\prime} / t=0, \tilde{U}=1, V=2\right)$, and (c) $\sqrt{12} \times \sqrt{12}$ droplet phase $\left(t^{\prime} / t=0.5, \tilde{U}=1, V=2.5\right)$. The dashed lines represent the band structures in the noninteracting limit.

whole phase diagram which supports a nonzero $\left\langle\tau^{z}\right\rangle$. $\mathrm{PCO}_{\mathrm{III}}$ instead has a metallic character. In the limit $t^{\prime}=-t$, shown in Fig. 4(c), it is a semimetal with bands touching at the $\Gamma$ and $K$ points.

If one interprets as usual the $120^{\circ}$ ordering as the mean-field equivalent of a Mott insulating phase, it can be argued that the PCO is a neat example where charge ordering enhances the effect of Hubbard-type interactions, which happens because the reconstruction of the band structure leads to half-filled bands that are much narrower than in the homogeneous phase, as these rapidly shrink upon increasing $V$ [Figs. 5(a) and 5(b)]. One notable example of this positive interplay between charge ordering and the Mott mechanism is found in the dichalcogenide $\mathrm{TaS}_{2}[18,19]$ (although the charge-ordering pattern there has a larger periodicity of 13 sites per cell, as discussed below).

(d) $\sqrt{12} \times \sqrt{12}$ droplet phase. For $t^{\prime} / t>0.1$, an additional phase emerges in the small- $\tilde{U}$ regime, located between the two charge-ordered phases found at small and large $V$. This phase, which is stabilized with respect to the PCO phase by purely kinetic effects [37], has a large periodicity with 12 sites in the unit cell. It is detected by the coexistence of peaks in the charge-charge correlation function at the $K$ points (characterizing threefold order) plus all the $M$ (whose combination describes a phase with fourfold symmetry [38]) and $K / 2$ points. The latter are absent in the phases with pure threefold symmetry. The $M$-point correlation is shown in Fig. 2(c). The real-space snapshot in Fig. 3(c) shows that the charges form disconnected hexagonal droplets, composed of essentially doubly occupied sites. These are surrounded by sites which are either empty or almost empty. Correspondingly, this phase has a strongly insulating character due to the opening of a gap $\propto V$ at the Fermi energy [Fig. 5(c)]. This phase also displays weak orbital order on the hexagons, signaled by nonzero orbital correlations at points $M$ and $K / 2$.

We note that the emergence of a charge pattern with a highorder periodicity such as the one found here is not at all trivial given that the electron-electron repulsion is restricted to only nearest-neighbor sites. The region in parameter space where the $\sqrt{12} \times \sqrt{12}$ droplet phase is stabilized actually includes the limit $t^{\prime}=t$ where Eq. (1) becomes equivalent to the spinful extended Hubbard model, but it was overlooked in previous studies [7,38-42]. Interestingly, the present 12-site period is very close to the 13-site star of David modulation found in the triangular dichalcogenide compound $1 T-\mathrm{TaS}_{2}$ [19]. The hexagonal droplets in Fig. 3(c) are also similar to those recently predicted theoretically in the kagome lattice at $n=1 / 3$ filling $[34,43]$, but in that case they are a natural consequence of the larger unit cell of the underlying lattice (see also Refs. [25,44]).

Finally, we mention that the $\sqrt{12} \times \sqrt{12}$ phase obtained in the narrow interval $-1 / 3<t^{\prime} / t \lesssim-0.1$ [see Fig. 2(b)] has the doubly occupied hexagons replaced by empty sites and vice versa and can therefore be considered the dual to the droplet phase described above. The two are separated by a phase with coexisting incommensurate charge and orbital order, denoted C/ODW.

(e) Homogeneous orbitally ordered phases. Several phase transitions are also found within the charge homogeneous region at low $V$. The system evolves upon increasing the effective local interaction from a paraorbital metal at small $\tilde{U}$ to a spiral $120^{\circ}$ orbitally ordered insulating phase at large $\tilde{U}$ (with the orbital moments arranged in planes perpendicular to the lattice as soon as $t^{\prime} \neq t$ ), going through an intermediate region with more complex orderings, denoted as ODW in Fig. 2(a). Although a precise study of this intermediate region is beyond the scope of the present work, we would like to stress the following points: (i) Within our real-space unrestricted approach, we have recovered the two intermediate mean-field phases of the spinful Hubbard model [35,36], namely, an incommensurate orbital (spin) ordered phase whose wave vector evolves with $\tilde{U}$ and a zigzag stripe compatible with the points $k=( \pm \pi, 0)$. (ii) While previous studies have looked for solutions breaking the symmetry at a single wave vector, we find solutions compatible with two or more coexisting $k$ vectors, possibly a mean-field indication of a tendency to a structureless orbital liquid state. (iii) Away from the case of the isotropic Hubbard model, i.e., as soon as $t^{\prime} \neq t$, the zigzag stripe phase seems to disappear, and only the incommensurate regions with coexisting order survive. (iv) At $t^{\prime}=0.5$, the critical parameter $\tilde{U}_{c}$ is decreased by $25 \%$ with respect to the isotropic case $t^{\prime}=t$, in agreement with the corresponding reduction of the bandwidth. 

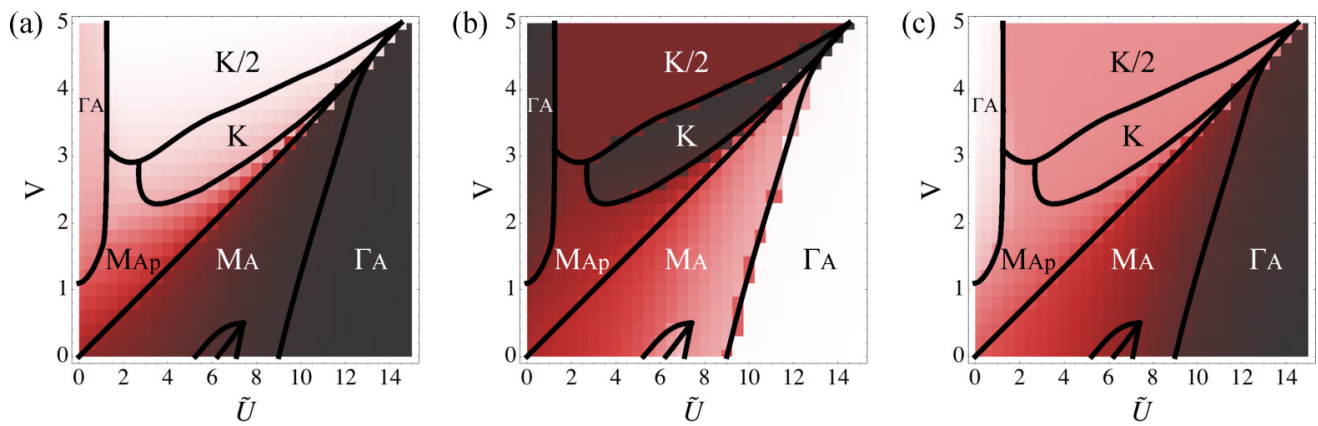

FIG. 6. (Color online) Phase diagrams obtained from ED on a 12-site cluster at fixed $t^{\prime}=0.5 t$. (a) Charge-charge correlation $C(K)$. (b) Orbital-orbital correlation $T(K)$. (c) Expectation value of the double occupation $D$. The color maps are in arbitrary scale, increasing from dark to bright. On each panel are shown the ground-state symmetry sectors (translation and point group) and first-order transition lines (thick lines) reflecting symmetry breaking driven either by the charges, the orbitals or both. The symmetry sectors in the small dome at low $V$ is respectively $K / 2$ and $M_{A_{p}}$ as $\tilde{U}$ increases.

\section{Exact diagonalization}

To ascertain if the variety of phases found at mean-field level in Fig. 2(a) is robust against quantum fluctuations, we have performed a systematic study of the model equation (1) via Lanczos diagonalization on a 12 -site cluster. Such a cluster is compatible with all the symmetries of the expected chargeordered phases except for $\mathrm{PCO}_{\mathrm{I}}$, which has a nine-site unit cell due to the presence of additional $120^{\circ}$ orbital order. It is also compatible with the orbital orders found by UHF in the low- $V$ region, except for the $( \pm \pi, 0)$ zigzag stripes and the incommensurate stripes characteristic of intermediate $\tilde{U}$.

Figure 6 shows the phase diagram obtained in the $(U, V)$ plane for $t^{\prime}=0.5 t$ by combining the symmetry character of the ground state for the charge-charge [Fig. 6(a)] and the orbitalorbital [Fig. 6(b)] correlations at the corner of the Brillouin zone $(K)$ and the average double occupation [Fig. 6(c)]. Our ED results for small clusters confirm that the very rich physical picture created by the interplay between charge and orbital degrees of freedom persists even beyond the mean-field level. A large number of domains with different symmetries are obtained, separated by first-order transitions, as displayed in each panel of Fig. 6; these domains can be associated with all of the different phases found in Fig. 2(a).

(a) Charge-ordered phases. The buildup of $K$-point charge correlations in Fig. 6(a), which is expected in all the chargeordered phases described in Sec. III B, shows remarkable agreement with the UHF charge-ordering transition lines reported in Fig. 2(a) and asymptotically follows the analytical prediction $V_{c}=\tilde{U} / 3$. We provide in Table I some representative values of $C(k)$ at points $K$ and $M$ for the different charge-ordered phases, as computed numerically by both UHF and ED and analytically on ideal electrostatic patterns. As one can see, a quantitative agreement is found between the two numerical methods, which allows for direct identification of the mean-field phases in the ED results. From Fig. 6 and Table I, we associate the different charge-ordered phases as follows: $K / 2$ and $K \rightarrow \mathrm{PCO}, M_{A_{p}} \rightarrow \mathrm{PL}$, and $\Gamma_{A} \rightarrow \sqrt{12} \times \sqrt{12}$.

First, we identify both the $K / 2$ and $K$ ground states with the broad PCO region of Fig. 2(a). We attribute the additional transition seen here, which is absent in UHF, to the fact that the ninefold orbital order present in the $\mathrm{PCO}_{\mathrm{I}}$ phase is not compatible with the 12-site cluster, so that different orderings are stabilized instead. This may due to either the exact treatment of the correlations or the size of the lattice. Accordingly, this change of symmetry is not observable in $C(K)$, but it is clearly seen in $T(K)$ in Fig. 6(b).

Second, by looking at the charge correlations in real space (not shown), it is possible to associate the $M_{A_{p}}$ ground state with PL order. The PL is found here instead of the IPL expected from UHF because the considered cluster is too small to capture the subtle kinetic effects which distinguish between these two phases. This is confirmed by the fact that the PL is also selected in the UHF solution when a 12-site cluster is considered, as we have checked.

Third, the $\sqrt{12} \times \sqrt{12}$ droplet phase found in the mean field can be associated with the $\Gamma_{A}$ ground state at low $\tilde{U}$ in Fig. 6. This phase has a charge signature corresponding to a mixture of the high-symmetry $k$ vectors $K, M$, and $K / 2$, with dominant weight on the first two, which agrees with the UHF and analytical results (see Table I). Also, it is in this phase that we find the strongest double occupancy [Fig. 6(c)], which corresponds to the doubly occupied sites on the hexagons in Fig. 3(c). Our ED results confirm the finding that the $\sqrt{12} \times \sqrt{12}$ droplet phase remains stable in the spinful extended Hubbard model $\left(t^{\prime}=t\right)$.

(b) Orbitally ordered phases. The different phases found in UHF upon increasing $\tilde{U}$ at low $V$ also have their direct

TABLE I. Comparison of the charge-charge correlation $C(k) / N$ for the high-symmetry points $K$ and $M$ in the different charge-ordered phases, computed analytically on the ideal patterns, via UHF and ED. The values for PCO, IPL/PL, and $\sqrt{12} \times \sqrt{12}$ correspond to $t^{\prime} / t=0.5$ and $(\tilde{U}, V)$ of $(6,4),(1,1.2)$, and $(0,3)$, respectively.

\begin{tabular}{lcccc}
\hline \hline & Point & PCO & IPL/PL & $\sqrt{12} \times \sqrt{12}$ \\
\hline Analytical & $K$ & $1 / 3$ & $1 / 4$ & $1 / 4$ \\
& $M$ & 0 & 0 & $1 / 9$ \\
UHF & $K$ & 0.302 & 0.121 & 0.227 \\
& $M$ & 0 & 0 & 0.065 \\
ED & $K$ & 0.308 & 0.157 & 0.262 \\
& $M$ & 0.006 & 0.055 & 0.063 \\
\hline \hline
\end{tabular}


analogs in ED. We associate the spiral $120^{\circ}$ phase obtained in UHF with the $\Gamma_{A}$ phase at large $\tilde{U}$ and small $V$ : here the double occupation is strongly suppressed, indicating a Mott insulating state with large threefold orbital correlations compatible with such an order $[45,46]$. We note that the critical value for the Mott transition, $U_{c} \approx 9 t$, is smaller than the value $U_{c} \approx 12 t$ reported from analogous ED calculations in the isotropic case [45], which can be understood by observing that the bandwidth is reduced by roughly $25 \%$ for the considered $t^{\prime}=0.5 t$ (Fig. 1). We also observe a small dome in the middle of the $M_{A}$ phase, which we tentatively associate with the ODW orders obtained by UHF on larger systems, as suggested by the signatures in $T(K)$. In this dome, two distinct symmetry sectors corresponding to two different phases are encountered as $\tilde{U}$ increases, $K / 2$ and $M_{A_{p}}$, respectively.

\section{CONCLUSIONS}

We have considered a minimal electronic model which describes the interplay between frustrated electron-electron interactions and multiorbital effects on the half-filled triangular lattice. Our results based on the combination of a fully unrestricted Hartree-Fock method, which provides an accurate description of the multiband kinetic properties, and exact diagonalization on small clusters, which properly takes into account interorbital and intersite correlation effects, reveal a very rich phase diagram. A number of original charge-ordered and orbitally ordered phases are displayed, whose occurrence can be tuned by varying the multiorbital band structure parameters and which could be relevant to a variety of $d$-electron systems on the triangular lattice. These include threefold metallic charge-ordered phases such as the pinball liquid, which was originally predicted to occur in quarter-filled lattices and is shown here to be stable at half filling, as well as its dual, the inverse pinball liquid. Both these phases could be relevant to $\mathrm{AgNiO}_{2}$, where a robust threefold charge-ordered metal has been experimentally observed $[15,16]$, which we therefore associate with a pinball state (see also Ref. [24]). An original insulating droplet phase with a large $\sqrt{12} \times \sqrt{12}$ periodicity is also obtained here, which was overlooked in previous studies of the extended Hubbard model and which could be closely related to the star of David charge-ordered phase of the triangular dichalcogenide compound $1 T-\mathrm{TaS}_{2}$ [19]. The insulating threefold PCO phase obtained here could also find a possible experimental realization, as suggested in triangular absorbate layers [47]. All these possible connections with the experiments emphasize the general nature of our study and should motivate further investigations in stabilizing original charge orders driven by frustrated electronic interactions and kinetic effects.

Finally, we note that in this paper we have restricted our study to a perfectly stoichiometric case where the $e_{g}$ doublet is initially quarter filled, corresponding to an average valence $\mathrm{Ni}^{3+}$ in $\mathrm{AgNiO}_{2}$. It has been proposed, however, that the filling of the $e_{g}$ levels in nickelates might actually differ from this value $[48,49]$, as extra electrons could be transferred from the oxygen $2 p$ orbitals provided that the electrostatic cost $\tilde{U}=U-3 J_{H}$ on the Ni sites is sufficiently low $[33,50]$. The very observation of a pinball state in $\mathrm{AgNiO}_{2}$ indicates that the effective interaction $\tilde{U}$ is low in this material [see Fig. 2(a)], and the existence of a "negative charge transfer" from the oxygen atoms is indeed compatible with the estimates of Ref. [16], which indicate a reduced average valence of $2.85+$ per Ni. Studying the present model away from the perfectly quarter-filled configuration will certainly be of interest in view of these considerations.

\section{ACKNOWLEDGMENTS}

The authors thank J. Merino and L. de' Medici for useful discussions. This work is supported by the French National Research Agency through Grants No. ANR-12-JS040003-01 SUBRISSYME and No. ANR-2010-BLANC-0406-0 NQPTP.
[1] C. Hotta and N. Furukawa, Phys. Rev. B 74, 193107 (2006).

[2] M. Kaneko and M. Ogata, J. Phys. Soc. Jpn. 75, 014710 (2006).

[3] M. Miyazaki, C. Hotta, S. Miyahara, K. Matsuda, and N. Furukawa, J. Phys. Soc. Jpn 78, 014707 (2009).

[4] L. Cano-Cortés, J. Merino, and S. Fratini, Phys. Rev. Lett. 105, 036405 (2010).

[5] L. Cano-Cortés, A. Ralko, C. Février, J. Merino, and S. Fratini, Phys. Rev. B 84, 155115 (2011).

[6] J. Merino, A. Ralko, and S. Fratini, Phys. Rev. Lett. 111, 126403 (2013).

[7] L. F. Tocchio, C. Gros, X.-F. Zhang, and S. Eggert, Phys. Rev. Lett 113, 246405 (2014).

[8] M. Udagawa and Y. Motome, Phys. Rev. Lett 98, 206405 (2007).

[9] K. Kuroki, Sci. Technol. Adv. Mater. 10, 024312 (2009).

[10] S. Mahmoudian, L. Rademaker, A. Ralko, S. Fratini, and V. Dobrosavljević, arXiv:1412.4441.

[11] K. Takada, H. Sakurai, E. Takayama-Muromachi, F. Izumi, R. A. Dilanian, and T. Sasak, Nature (London) 422, 53 (2003).
[12] N. P. Ong and R. J. Cava, Science 305, 52 (2004).

[13] M.-H. Julien, C. de Vaulx, H. Mayaffre, C. Berthier, M. Horvatić, V. Simonet, J. Wooldridge, G. Balakrishnan, M. R. Lees, D. P. Chen, C. T. Lin, and P. Lejay, Phys. Rev. Lett. 100, 096405 (2008).

[14] H. Alloul, I. R. Mukhamedshin, A. V. Dooglav, Ya. V. Dmitriev, V.-C. Ciomaga, L. Pinsard-Gaudart, and G. Collin, Phys. Rev. B 85, 134433 (2012).

[15] E. Wawrzyńska, R. Coldea, E. M. Wheeler, I. I. Mazin, M. D. Johannes, T. Sörgel, M. Jansen, R. M. Ibberson, and P. G. Radaelli, Phys. Rev. Lett. 99, 157204 (2007).

[16] G. L. Pascut, R. Coldea, P. G. Radaelli, A. Bombardi, G. Beutier, I. I. Mazin, M. D. Johannes, and M. Jansen, Phys. Rev. Lett. 106, 157206 (2011).

[17] H. Yoshida, Y. Muraoka, T. Sörgel, M. Jansen, and Z. Hiroi, Phys. Rev. B 73, 020408(R) (2006).

[18] L. Perfetti, P. A. Loukakos, M. Lisowski, U. Bovensiepen, H. Berger, S. Biermann, P. S. Cornaglia, A. Georges, and M. Wolf, Phys. Rev. Lett. 97, 067402 (2006). 
[19] B. Sipos, A. F. Kusmartseva, A. Akrap, H. Berger, L. Forró and E. Tutiš, Nat. Mater. 7, 960 (2008).

[20] J. Kanamori, Prog. Theor. Phys. 30, 275 (1963).

[21] L. de' Medici, J. Mravlje and A. Georges, Phys. Rev. Lett. 107, 256401 (2011).

[22] L. de' Medici, Phys. Rev. B 83, 205112 (2011).

[23] A. Georges, L. de' Medici, and J. Mravlje, Annu. Rev. Condens. Matter Phys. 4, 137 (2013).

[24] A. Ralko, J. Merino, and S. Fratini, Phys. Rev. B 91, 165139 (2015).

[25] W. Koshibae and S. Maekawa, Phys. Rev. Lett. 91, 257003 (2003).

[26] H. Alloul, I. R. Mukhamedshin, T. A. Platova, and A. V. Dooglav, Europhys. Lett. 85, 47006 (2009).

[27] J. C. Slater and G. F. Koster, Phys. Rev. 94, 1498 (1954).

[28] G. Jackeli and G. Khaliullin, Phys. Rev. Lett. 101, 216804 (2008).

[29] F. Vernay, K. Penc, P. Fazekas, and F. Mila, Phys. Rev. B 70, 014428 (2004).

[30] J.-S. Kang, S. S. Lee, G. Kim, H. J. Lee, H. K. Song, Y. J. Shin, S. W. Han, C. Hwang, M. C. Jung, H. J. Shin, B. H. Kim, S. K. Kwon, and B. I. Min, Phys. Rev. B 76, 195122 (2007).

[31] T. Sörgel and M. Jansen, J. Solid State Chem. 180, 8 (2007).

[32] H. Uchigaito, M. Udagawa, and Y. Motome, J. Phys. Soc. Jpn. 80, 044705 (2011).

[33] I. I. Mazin, D. I. Khomskii, R. Lengsdorf, J. A. Alonso, W. G. Marshall, R. M. Ibberson, A. Podlesnyak, M. J. MartínezLope, and M. M. Abd-Elmeguid, Phys. Rev. Lett. 98, 176406 (2007).

[34] K. Ferhat and A. Ralko, Phys. Rev. B 89, 155141 (2014).

[35] H. R. Krishnamurthy, C. Jayaprakash, S. Sarker, and W. Wenzel, Phys. Rev. Lett. 64, 950 (1990).
[36] C. Jayaprakash, H. R. Krishnamurthy, S. Sarker, and W. Wenzel, Europhys. Lett. 15, 625 (1991).

[37] It can be shown straightforwardly that the potential energies of the droplet and PCO phases coincide when $\tilde{U}=0$ (the cost due to $V$ being exactly equal), while the droplet phase is rapidly destabilized by $\tilde{U}$ due to the larger double occupation.

[38] B. Davoudi, S. R. Hassan, and A.-M. S. Tremblay, Phys. Rev. B 77, 214408 (2008).

[39] G. Santoro, S. Scandolo, and E. Tosatti, Phys. Rev. B 59, 1891 (1999).

[40] K. Kanada, T. Watanabe, S. Onari, and Y. Tanaka, J. Phys. Chem. Sol. 69, 3334 (2008)

[41] J. Gao and J. Wang, J. Phys. Condens. Matter 21, 485702 (2009).

[42] S. R. Hassan and L. de Medici, Phys. Rev. B 81, 035106 (2010).

[43] F. Pollmann, K. Roychowdhury, C. Hotta, and K. Penc, Phys. Rev. B 90, 035118 (2014).

[44] O. E. Peil, A. Georges, and F. Lechermann, Phys. Rev. Lett. 107, 236404 (2011).

[45] M. Capone, L. Capriotti, F. Becca, and S. Caprara, Phys. Rev. B 63, 085104 (2001).

[46] P. Sahebsara and D. Sénéchal, Phys. Rev. Lett. 100, 136402 (2008).

[47] R. Cortés, A. Tejeda, J. Lobo-Checa, C. Didiot, B. Kierren, D. Malterre, J. Merino, F. Flores, E. G. Michel, and A. Mascaraque, Phys. Rev. B 88, 125113 (2013).

[48] A. Demourgues, F. Weill, B. Darriet, A. Wattiaux, J. Grenier, P. Gravereau, and M. Pouchard, J. Solid State Chem. 106, 330 (1993).

[49] T. Mizokawa, D. I. Khomskii, and G. A. Sawatzky, Phys. Rev. B 61, 11263 (2000).

[50] A. Subedi, O. E. Peil, and A. Georges, Phys. Rev. B. 91, 075128 (2015). 